\title{
Auswirkungen von touristischen Infrastrukturinvestitionen auf die Einkommenslage im Berggebiet
}

\section{Einleitung}

Im Zusammenhang mit touristischer Entwicklung werden oft zwei irrige Meinungen vertreten. Einerseits wird behauptet, eine von der Abwanderung betroffene Berggemeinde brauche sich nur um die touristische Entwicklung zu bemühen, um einer sorgenfreien Zukunft entgegenzugehen. Dazu müsse lediglich ein Skilift erstellt werden, notfalls - bei geringer allgemeiner Attraktivität - auch ein Hallenbad. Anderseits wird dieser Behauptung entgegengesetzt, daß viele Bergbahnen und praktisch alle Hallenbäder defizitär seien.

Beide Aussagen besitzen ein Körnchen Wahrheit, doch müssen die Probleme schon etwas differenzierter betrachtet werden.

Nicht jeder Flecken in unseren Berggebieten eignet sich a priori für Erholung und Fremdenverkehr. Damit eine touristische Erschließung langfristig auch Erfolg haben kann, ist eine ganze Reihe von Voraussetzungen notwendig. Sie seien hier nur kurz und sicher unvollständig genarint:

Attraktive Landschaft, vielseitige Ski- und/oder Wandergebiete, genügendes Arbeitskräftepotential, gute Erreichbarkeit von den Agglomerationen aus, eine gewisse vorhandene touristische und Beherbergungsinfrastruktur, keine Konkurrenz in unmittelbarer Nachbarschaft, die über Kapazitätsreserven verfügt. Von besonderem Vorteil ist es auch, wenn der Name der Ortschaft oder der Region schon einen gewissen Bekanntheitsgrad aufweist.

Es stimmt zwar, daß viele Bergbahnen und Skilifte sich in einem mehr oder weniger ständigen Kampf mit den roten Zahlen befinden und bei Hallenbädern - vor allem bei steigenden Energiekosten - die Ausgaben die Einnahmen übersteigen. Trotzdem aber läßt sich der Bau solcher Tourismusanlagen volkswirtschaftlich durchaus rechtfertigen, da die dadurch induzierten Einkommen in andern Betrieben der Region die Verluste um ein Vielfaches übertreffen können.

Am Beispiel des Bezirkes Oberhasli soll hier aufgezeigt werden, daß eine Bergbahn wie die MeiringenHasliberg Bahnen die Einkommenslage und Lebensqualität der Bergbevölkerung weitgehend positiv beeinflussen kann. Dabei ist zwischen der erzielten Attraktivitätssteigerung als langfristigem Effekt und der Bauphase mit zeitlich begrenzter Einkommenswirkung zu unterscheiden. Es handelt sich hier auch um ein Gebiet, bei welchem sämtliche oben aufgeführten Voraussetzungen für eine erfolgreiche touristische Entwicklung durchaus erfüllt sind.

Die Elektrowatt Ingenieurunternehmung AG in Zürich wurde 1978 vom Schweizerischen Nationalfonds mit einer Studie im Rahmen des Forschungsprogrammes «Regionalprobleme in der Schweiz» über die Auswirkungen von Großinvestitionen auf die Einkommenslage im Berggebiet beauftragt. Untersucht wurden die drei Fallstudien Albula-Landwasser Kraftwerke, Bernhardin Straßentunnel und die Meiringen-Hasliberg Bahnen (MHB), wobei nur bei diesem dritten Projekt neben der Bau- auch die Betriebsphase einbezogen wurde.

\section{Projekt und Projektgebiet}

Das Haslital kann als traditionelle touristische Region bezeichnet werden, war es doch schon Ende des 19. Jahrhunderts ein bekanntes Sommerferiengebiet. Nach den Krisenjahren, in denen viele Gastgewerbebetriebe aufgegeben werden mußten und auch die Bevölkerungszahl ihren Tiefststand erreichte, setzte wiederum eine deutliche touristische Entwicklung ein. Mit der Eröffnung der Gondelbahn Hasliberg-Käserstatt im Jahre 1960 fand auch der Wintersport auf dem Hasliberg Eingang. Diese Anlage wurde zwar durch Skilifte erweitert, doch erst zusammen mit den durch die MHB erschlossenen Skipisten entstand ein ausgedehntes, sehr vielfältiges Skigebiet, das in den oberen Partien auch für den Frühlingsskilauf ausgezeichnet geeignet ist. Zudem entstand für die Bevölkerung des Haslibergs mit der Sektion Meiringen-Reuti eine direkte Talverbindung.

Im November 1969 suchten die Gemeinden Meiringen und Hasliberg, mit Unterstützung der Gemeinden Schattenhalb, Innertkirchen, Guttannen, Gadmen und der Bäuertgemeinde (Alpkorporation) Hasliberg beim Eidgenössischen Verkehrs- und

Dr. Christian Oswald, Elektrowatt Ingenieurunternehmung AG, 8022 Zürich und Dr. Beatrix Elsasser-Rusterholz, 8942 Oberrieden. 
Energiedepartement um eine Konzession nach, die im Oktober 1970 erteilt wurde.

Die Aktiengesellschaft für den Bau und Betrieb der MHB wurde 1972 gegründet und der Betrieb nach nur 10 Monaten Bauzeit am 30. Dezember 1973 auf den Sektionen Meiringen-Reuti (Luftseilbahn), Reuti-Bidmi und Bidmi-Mägisalp (Gondelbahnen) sowie Mägisalp-Planplatten (Sesselbahn) aufgenommen. Bis Ende 1978 wurde die Anlage um den Sessellift Bidmi-Käserstatt und den Skilift Spycher erweitert. Mit dem Sessellift Bidmi-Käserstatt wurde die Verbindung zum Skigebiet, das durch die Gondelbahn Hasliberg-Käserstatt mit den zugehörigen Skiliften erschlossen wird, geschaffen.

\section{Die Einkommenswirkungen während der Bauphase der MHB}

Anhand der Bauabrechnung wurde zuerst die primäre Zahlungsinzidenz ermittelt. Dabei wurden folgende drei Zonen für die Zahlungsempfänger unterschieden:

- Projektgebiet: umfaßt die sechs Gemeinden, welche das Konzessionsgesuch stellten.

- Projektregion: übrige Planungsregion OberlandOst.

- übrige Schweiz.

(Zahlungen ins Ausland wurden keine geleistet.)

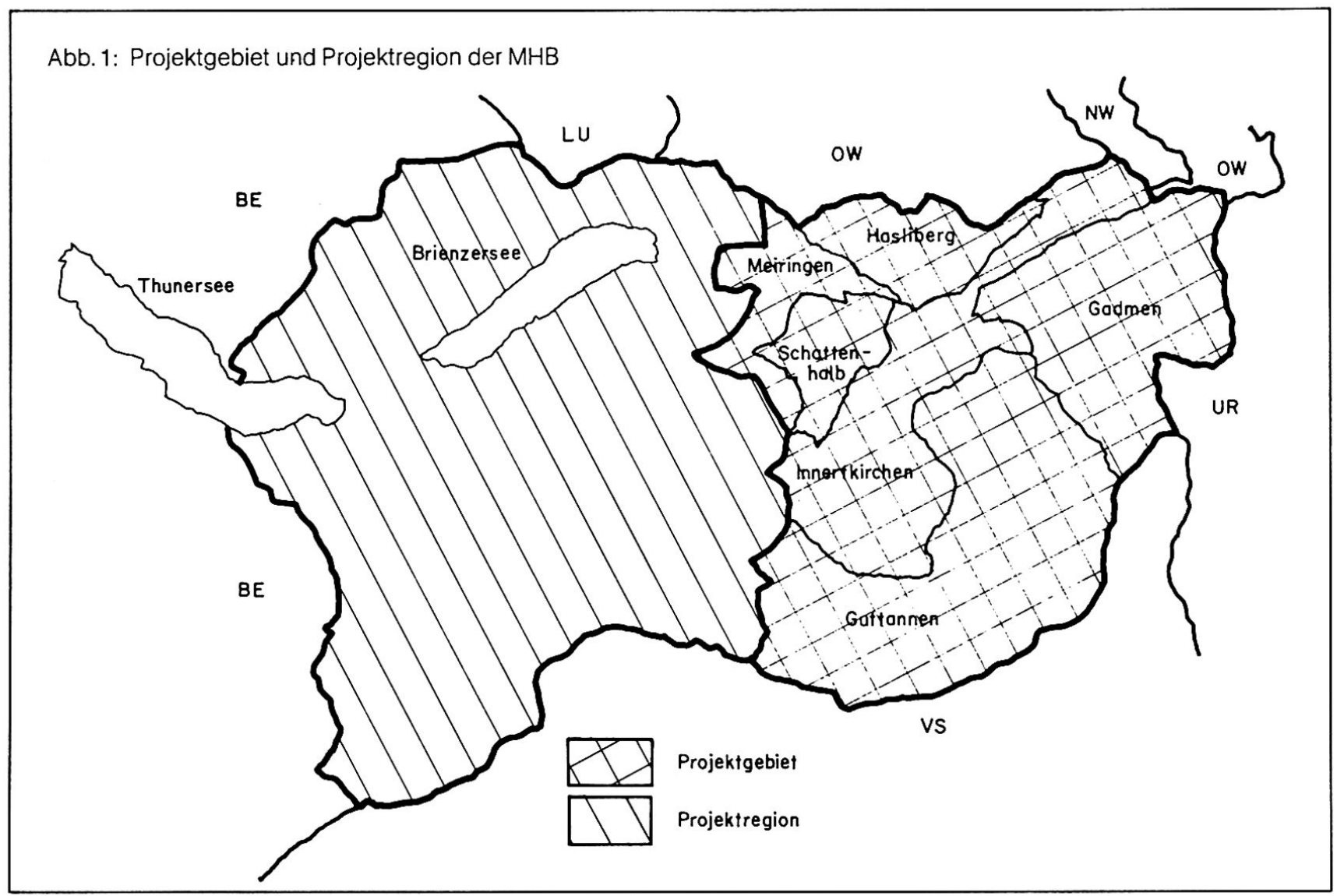

Ein Drittel aller Zahlungen floß ins Projektgebiet, währenddem die Projektregion am Bau nur unwesentlich beteiligt war:

Tabelle 1: Hauptergebnisse der primären Zahlungsinzidenz

\begin{tabular}{lrlllll}
\hline $\begin{array}{l}\text { Inzidenzgebiet } \\
\text { (Anzahl Einwohner) }\end{array}$ & \multicolumn{2}{l}{$\begin{array}{l}\text { Anzahl Zahlungs- } \\
\text { empfänger }\end{array}$} & $\begin{array}{l}\text { Gesamtwert der } \\
\text { Zahlungen in } \\
\text { in 1000 Fr. }\end{array}$ & $\begin{array}{l}\text { Durchschnittswert } \\
\text { pro Zahlungs- } \\
\text { empfänger in Fr. }\end{array}$ & $\begin{array}{l}\text { Durchschnittswert } \\
\text { pro Einwohner } \\
\text { in Fr. }\end{array}$ \\
\hline Projektgebiet (7821) & 83 & $(55,7 \%)$ & 5870 & $(33,1 \%)$ & 70726 & 750.60 \\
Übrige Projektregion (25160) & 15 & $(10,1 \%)$ & 493 & $(2,8 \%)$ & 32881 & 19.60 \\
Übrige Schweiz (6236802) & 51 & $(34,2 \%)$ & 11348 & $(64,1 \%)$ & 222503 & 1.80 \\
\hline Total (6269783) & 149 & $(100 \%)$ & 17711 & $(100 \%)$ & 118867 & 2.80 \\
\hline
\end{tabular}


In der ganzen Region Oberland-Ost wurden zum größten Teil Firmen aus der Bauwirtschaft und verwandten Branchen berücksichtigt (über $85 \%$ ), währenddem die elektromechanische Ausrüstung, welche betragsmäßig sehr stark ins Gewicht fiel (42\%), von auswärts geliefert wurde.

Zur Abschätzung der direkten Einkommenswirkung, die während der Bauphase vom Investitionsprojekt auf dessen Umgebung ausgegangen ist, wird zwischen dem Gewinneinkommen der einheimischen Unternehmer und dem Lohneinkommen der einheimischen ${ }^{1)}$ Arbeitnehmer unterschieden.

Bei der Schätzung des Gewinneinkommens geht man von den 5,9 Mio Franken aus, welche als Zahlungen vom Projekt ins Projektgebiet flossen. Zudem wurden noch Betonmaterial und Holz im Wert von etwa 3 Mio Franken aus dem Projektgebiet selbst bezogen. Von den Umsatzzahlen von etwa 8,9 Mio Franken beträgt der zusätzliche Umsatz, der ohne Projekt nicht erzielt worden wäre, nach Schätzungen von Fachleuten etwa 8,5 Mio Franken oder $96 \%$ vom Umsatz. Der von den Unternehmern durch diesen zusätzlichen Umsatz zusätzlich erzielte $\mathrm{Ge}$ winn dürfte etwa 2,5 Mio Franken ausmachen.

Die Bedeutung der zusätzlichen Lohneinkommen im Projektgebiet darf nicht überschätzt werden. Sie betragen schätzungsweise 200000 Franken. Die einheimischen Baugeschäfte und Handwerksbetriebe konnten ihre Arbeiten ohne zusätzliche ständige Arbeitskräfte ausführen. Das zusätzliche Lohneinkommen entfiel mehrheitlich auf nur temporär beschäftigte Bergbauern.

Bei der direkten Einkommenswirkung dürfen die Lohneinkommen der auswärtigen Arbeitskräfte nicht berücksichtigt werden, da sie hier nicht niedergelassen und somit auch nicht steuerpflichtig waren. Bei der indirekten Einkommenswirkung müssen jedoch diese Einkommen auch berücksichtigt werden, da sowohl Pendler wie Wochenaufenthalter im Projektgebiet Ausgaben tätigten.

Die direkte Einkommenswirkung während der Bauphase betrug im Total 2,7 Mio Franken, was etwa $15 \%$ der Bausumme ausmacht.

Diese 2,7 Mio Franken zusätzlicher Einkommen erlaubten es der Bevölkerung, ihre Konsumausgaben zu erhöhen. Praktisch die ganze indirekte Einkommenswirkung geht auf solchen zusätzlichen Konsum zurück, und zwar von Gütern und Dienstleistungen, die im Projektgebiet selbst produziert bzw. erbracht wurden. Das zusätzliche Einkommen, das die einheimischen Produzenten und Dienstleistungsbetriebe dadurch erzielen, beträgt im Projektgebiet schätzungsweise $28 \%$. Es wird davon ausgegangen, daß 70\% der zusätzlichen Einkommen für den loka-

1) Auswärtige Firmen ließen ihre Arbeiten durch Wochenaufenthalter und Pendler ausführen, so daß während des Baues keine zusätzlichen auswärtigen Arbeitnehmer im Projektgebiet niedergelassen waren. len Konsum aufgewendet werden, wobei der lokale Wertschöpfungsanteil vom Gesamtkonsum etwa $40 \%$ ausmacht. Die indirekte Einkommenswirkung beträgt somit etwa 760000 Franken.

Ähnliche Schätzungen und Berechnungen kann man auch bei den Ausgaben der Wochenaufenthalter und Pendler anstellen und kommt dabei auf eine indirekte Einkommensauswirkung von 93000 Franken bzw. von 3000 Franken.

Die indirekte Einkommenswirkung beträgt im Total schätzungsweise 860000 Franken, jedoch nur für die erste Ausgabenperiode. In der Folge wird dieses zusätzliche Einkommen jedoch wiederum für Güter und Dienstleistungen aus dem Projektgebiet ausgegeben. Dieser Prozeß kann sich theoretisch beliebig lange fortsetzen - mit immer kleineren Werten. Die Summe sämtlicher indirekter Einkommenswirkungen, welche aus der Literatur als Multiplikatoreffekt bekannt ist, beläuft sich für alle folgenden Perioden auf rund $40 \%$ des indirekten Einkommens der ersten Ausgabenperiode. Entsprechend dem geschätzten Konsumverhalten der Bevölkerung und dem geschätzten Wertschöpfungsanteil im Projektgebiet kann mit einer Gesamtsumme von 1,2 Mio Franken indirekter Einkommenswirkungen gerechnet werden.

Durch den Bau der ersten Etappe der MeiringenHasliberg Bahnen, welche eine Bausumme von fast 18 Mio Franken umfaßte, wurde eine Gesamteinkommenswirkung im Projektgebiet von etwa 3,9 Mio Franken erzielt. Der größere Teil, nämlich 2,7 Mio Franken (fast 70\%) entfiel auf die direkten Einkommen. Die restlichen $30 \%$ oder 1,2 Mio Franken wurden indirekt durch die höheren Konsumausgaben erzielt.

Der Einkommenszuwachs im Baujahr 1973 betrug pro Kopf der Bevölkerung Fr. 508.- oder etwa 3\% des durchschnittlichen jährlichen Pro-Kopf-Einkommens.

\section{Die Einkommenswirkung durch den Betrieb der MHB}

Die Meiringen-Hasliberg Bahnen weisen in den drei Betriebsjahren 1975 bis 1977 immer einen positiven und wachsenden Cash-Flow von 8100 Franken 1975 bis 22500 Franken 1977 aus, doch resultierte infolge der hohen, gesetzlich vorgeschriebenen Abschreibungen von mehreren hunderttausend Franken immer ein Betriebsverlust; der allerdings von 729000 Franken im Jahr 1975 auf 200000 Franken im Jahr 1977 eingeschränkt werden konnte.

Obwohl immer noch ein Betriebsverlust zu verzeichnen ist, war der Betrieb der MHB gesamtwirtschaftlich gesehen von Anfang ein voller Erfolg, denn die Einkommenswirkung ist viel höher zu bewerten als das ungünstige buchhalterische Ergebnis. 
Ähnlich wie in der Bauphase wird hier wieder zwischen verschiedenen Arten der Einkommenswirkung unterschieden:

Unmittelbare Effekte: direkte Auswirkungen indirekte Auswirkungen

Induzierte Effekte: direkte Auswirkungen indirekte Auswirkungen.

Unmittelbare Effekte gehen von den MHB direkt aus, vorwiegend in Form von Löhnen an die Bahnangestellten. Bei den induzierten Effekten handelt es sich um solche, die nicht durch den Bahnbetrieb selbst, sondern durch andere Unternehmen des Tourismussektors erzielt werden. Das Gastgewerbe und andere Dienstleistungsbetriebe erreichen infolge des durch den Bahnbetrieb ausgelösten Touristenbooms höhere Umsätze. Zu diesen Begünstigten muß auch das Baugewerbe gezählt werden.

- Die direkten Auswirkungen beziehen sich wiederum auf die zusätzlichen Gewinn- bzw. Lohneinkommen, welche durch den Bahnbetrieb oder andere touristisch beeinflußte Unternehmen erzielt werden.

- Die indirekten Auswirkungen betreffen die lokale Wertschöpfung, die bei der Deckung des lokalen Konsums erzielt wird, der durch die zusätzlichen Gewinn- bzw. Lohneinkommen möglich wurde.

Die bedeutendste unmittelbare Auswirkung besteht in der Schaffung von 14 bis 16 Ganzjahresstellen, 11 bis 18 Wintersaisonarbeitsplätzen und 2 bis 5 Sommersaisonarbeitsplätzen bei den MHB. An Spitzentagen werden zudem bis zu 10 Aushilfskräfte angestellt. Dazu kommen die Angestellten des Bergrestaurants Mägisalp, das zwischen 12 und 18 Beschäftigte umfaßt.

Die unmittelbaren Auswirkungen von etwa 800000 Franken an Löhnen pro Jahr ergeben langfristig einen wichtigen Effekt, trug doch vor allem die saisonale Arbeitsmöglichkeit bei den Bahnen im Winter dazu bei, daß die Abwanderung weitgehend gestoppt werden konnte.

Andere, etwas weniger wichtige unmittelbare Auswirkungen umfassen weitere Aufwandposten, die aber schließlich zu einem unbekannten Teil irgendwann aus dem Projektgebiet hinausfließen: elektrischer Strom für etwa 150000 Franken, Verkehrswerbung und Beiträge von etwa 100000 Franken, Pacht und Mieten von etwa 63000 Franken (1977). Die Unterhaltsarbeiten an den Anlagen machen pro Jahr ungefähr 100000 Franken aus, werden aber von den auswärtigen Herstellern ausgeführt.

Daneben kann auch die Bevölkerung vom Bahnbetrieb direkt profitieren, indem an Landbesitzer im Pistenbereich Entschädigungen in Form einer Jahresfreikarte (Wert ca. Fr.600.-) abgegeben und indem Transporte für die Landwirtschaft gratis ausgeführt werden.
Insgesamt werden die unmittelbaren direkten Einkommenswirkungen der MHB und ihrer vorgelagerten Betriebe (z. B. Elektrizitätswerk) auf 600000 bis 700000 Franken geschätzt. Rechnet man auch hier mit dem Multiplikatoreffekt von 1,4 für die unmittelbaren indirekten Effekte, so kommt man zu einem Betrag von insgesamt etwa 900000 Franken (Preisbasis 1973).

Die induzierte Einkommenswirkung, die durch den touristischen Aufschwung in andern Wirtschaftszweigen ausgelöst wurde, ist sehr schwierig abzuschätzen, da die statistische Erfassung der Übernachtungen nur in der Hotellerie gesichert ist. Die Vermietung von Ferienwohnungen ist jedoch im ganzen Berner Oberland relativ sehr stark verbreitet, besonders auf dem Hasliberg, wo die Hotellerie auf den Zielverkehr dimensioniert ist und nicht von einem Durchgangsverkehr profitieren kann. Im Jahre 1972 gab es lediglich 160 Vermieter von Ferienwohnungen auf dem Hasliberg, nur 6 Jahre später waren es schon über 200.

Aufgrund der Kurtaxeneinnahmen der Gemeinden des Projektgebietes konnten die Übernachtungen in der Parahotellerie unter Berücksichtigung der jeweiligen Kurtaxenansätze grob berechnet werden. Im ganzen Projektgebiet hat die Parahotellerie einen Anteil von etwa der Hälfte an allen Übernachtungen. In den letzten Jahren hat sich nicht nur eine Umstrukturierung zwischen Parahotellerie und Hotellerie ergeben, sondern auch zwischen der Sommerund der Wintersaison. Währenddem das Total der Übernachtungen im Projektgebiet zwischen 1970 und 1978 um $20 \%$ auf rund 180000 angestiegen ist, sind es bei den Logiernächten im Winter rund $130 \%$ (73 000). Dafür ist die Zuwachsrate im Sommer negativ, die Logiernächte sind von 119000 auf 108000 zurückgegangen.

Auf dem Hasliberg allein entfielen 1970 nur etwa ein Drittel der Übernachtungen auf den Winter, heute sind es schon $41 \%$, wobei das Total der Übernachtungen noch leicht erhöht werden konnte.

Der Einfluß der MHB auf den Tourismus des Hasliberges kann geschätzt werden, wenn die langfristige Entwicklung der Hotellogiernächte des Hasliberges mit derjenigen des ganzen Berner Oberlandes verglichen wird. Dabei zeigt sich, daß der Hasliberg zwischen 1973 und 1978 eine Zuwachsrate der Logiernächte von $0,4 \%$ aufwies, währenddem das Berner Oberland einen Rückgang von 1,6\% in Kauf nehmen mußte. Man kann deshalb annehmen, daß der Unterschied von $2 \%$ den MHB zuzuschreiben ist, sofern der Hasliberg ohne MHB eine gleichsinnige Entwicklung durchgemacht hätte wie das gesamte Berner Oberland.

Man konnte berechnen, daß im Durchschnitt der Jahre 1974 bis 1978 die MHB etwa 30000 zusätzliche Übernachtungen induzierte, wozu noch schätzungsweise 35000 Tagesgäste kommen, die in den lokalen 
Gastgewerbsbetrieben konsumieren und andere Dienstleistungen in Anspruch nehmen (z. B. Skischule).

Bei total rund 65000 Besuchertagen, durchschnittlichen Ausgaben von Fr.39.- pro Tag und Person (ohne Bahnauslagen) kommt man auf Totalausgaben von 2,5 Mio Franken. Bei einem durchschnittlichen Wertschöpfungsanteil im Projektgebiet von $73 \%$ beträgt die induzierte Einkommenswirkung rund 1,85 Mio Franken.

Die Impulse, die vom Betrieb der MHB auf die Bau- wirtschaft ausgehen, können nur näherungsweise abgeschätzt werden, da nicht eruiert werden kann, welche Bauvorhaben auf die Inbetriebnahme der MHB zurückgeführt werden können. Im Mittel der siebziger Jahre dürften sie bei etwa 0,5 Mio Franken pro Jahr liegen und vorwiegend auf den lokalen Wertschöpfungsanteil der Hotel- und Restaurantumbauten zurückgehen.

Die Gesamtwirkung der Betriebsphase kann folgendermaßen zusammengefaßt werden:

Tabelle 2: Die Gesamtwirkung der Betriebsphase der MHB in Mio Franken

\begin{tabular}{llllc}
\hline Einkommenswirkung & direkt & indirekt & \multicolumn{2}{c}{$\begin{array}{c}\text { Total } \\
\text { absolut }\end{array}$} \\
\hline in \%
\end{tabular}

\section{Die Gesamtwirkung der Bau- und Betriebs- phase}

Die Untersuchung hat ergeben, daß der Bau der MHB bis Ende 1973 einen einmaligen Einkommenseffekt für das Projektgebiet von etwa 3,9 Mio Franken gebracht hat, und daß durch den Betrieb der MHB eine jährliche Totalwirkung von 4,4 Mio Franken erzielt wird. Der sozio-ökonomische Effekt liegt in der Schaffung von 130 Äquivalenten von Ganzjahresarbeitsplätzen, von denen ungefähr ein Viertel auf Saisonstellen entfällt, sowie in einer Trendumkehr des Bevölkerungsschwundes, einer starken Tertialisierung der Erwerbsstruktur (von $45 \%$ tertiär Erwerbstätige 1970 auf $65 \%$ 1978), sowie in einer gegenüber dem Kanton Bern überdurchschnittlichen Entwicklung des Volkseinkommens.
Es dürfte daher unbestritten sein, daß die Großinvestition der Meiringen-Hasliberg Bahnen durch ihren Bau und vor allem ihren Betrieb einen äußerst wertvollen Beitrag zur Berggebietsförderung im Projektgebiet leistet.

\section{Literatur}

OSWALD, CHR. unter Mitarbeit von ELSASSER, B. und GUICCIARDI, R.: Auswirkungen von Großinvestitionen auf die Einkommenslage im Berggebiet. NFP "Regionalprobleme in der Schweiz», Bericht Nr.4, Bern, Januar 1980. 